\title{
OPEN Dynamic changes in the brain protein interaction network correlates with progression of $\mathrm{A} \beta 42$ pathology in Drosophila
}

\author{
Harry M. Scholes ${ }^{1,7}$, Adam Cryar $^{1,7}$, Fiona Kerr ${ }^{2,3,7}$, David Sutherland ${ }^{1}$, Lee A. Gethings ${ }^{4}$, \\ Johannes P. C. Vissers ${ }^{4}$, Jonathan G. Lees ${ }^{1,6}$, Christine A. Orengo ${ }^{1 \bowtie}$, Linda Partridge ${ }^{2,5 \bowtie}$ \& \\ Konstantinos Thalassinos ${ }^{1 \bowtie}$
}

\begin{abstract}
Alzheimer's disease (AD), the most prevalent form of dementia, is a progressive and devastating neurodegenerative condition for which there are no effective treatments. Understanding the molecular pathology of AD during disease progression may identify new ways to reduce neuronal damage. Here, we present a longitudinal study tracking dynamic proteomic alterations in the brains of an inducible Drosophila melanogaster model of AD expressing the Arctic mutant A $\beta 42$ gene. We identified 3093 proteins from flies that were induced to express $A \beta 42$ and age-matched healthy controls using label-free quantitative ion-mobility data independent analysis mass spectrometry. Of these, 228 proteins were significantly altered by $A \beta 42$ accumulation and were enriched for AD-associated processes. Network analyses further revealed that these proteins have distinct hub and bottleneck properties in the brain protein interaction network, suggesting that several may have significant effects on brain function. Our unbiased analysis provides useful insights into the key processes governing the progression of amyloid toxicity and forms a basis for further functional analyses in model organisms and translation to mammalian systems.
\end{abstract}

Alzheimer's disease $(\mathrm{AD})$ is a progressive and devastating neurodegenerative disease that is the most prevalent form of dementia ${ }^{1}$. Symptoms initially present as episodic memory loss and subsequently develop into widespread cognitive impairment. Two brain lesions are pathological hallmarks of the disease: plaques and neurofibrillary tangles. Plaques are extracellular aggregates of amyloid beta $(\mathrm{A} \beta)^{2}$, whereas neurofibrillary tangles are intraneuronal aggregates of hyperphosphorylated tau ${ }^{3,4}$. In addition to these hallmarks, the AD brain experiences many other changes, including metabolic and oxidative dysregulation ${ }^{5,6}$, DNA damage ${ }^{7}$, cell cycle re-entry ${ }^{8}$, axon loss ${ }^{9}$ and, eventually, neuronal death ${ }^{6,10}$.

Despite a substantial research effort, no cure for $\mathrm{AD}$ has been found. Effective treatments are desperately needed to cope with the projected increase in the number of new cases as a result of longer life expectancy and an ageing population. Sporadic onset is the most common form of $\mathrm{AD}(\mathrm{SAD})$, for which age is the major risk factor. Familial AD (FAD) - a less common (<1\%), but more aggressive, form of the disease-has an early onset of pathology before the age of $65^{11}$. FAD is caused by fully penetrant mutations in the $A \beta$ precursor protein (APP) and two subunits-presenilin 1 and presenilin 2-of the $\mathrm{V}$-secretase complex that processes APP in the amyloidogenic pathway to produce $A \beta$. Whilst the exact disease mechanisms of AD are not yet fully understood, this has provided support for $A \beta$ accumulation as a key player in its cause and progression ${ }^{1}$. A $\beta 42-a 42$ amino acid variant of the peptide-is neurotoxic ${ }^{12}$, necessary for plaque deposition ${ }^{13}$ and sufficient for tangle formation ${ }^{14}$. The Arctic mutation in $\mathrm{A} \beta 42$ (Glu22Gly) ${ }^{15}$ causes a particularly aggressive form of familial AD that is associated with an increased rate and volume of plaque deposition ${ }^{16}$. Genetic analyses of SAD, however, suggest a complex molecular pathology, in which alterations in neuro-inflammation, cholesterol metabolism

\footnotetext{
${ }^{1}$ Institute of Structural and Molecular Biology, University College London, London, UK. ${ }^{2}$ Institute of Healthy Ageing, University College London, London, UK. ${ }^{3}$ Department of Biological and Biomedical Sciences, School of Health and Life Sciences, Glasgow Caledonian University, Glasgow, UK. ${ }^{4}$ Waters Corporation, Wilmslow, UK. ${ }^{5}$ Max Planck Institute for Biology of Ageing, Cologne, Germany. ${ }^{6}$ Present address: Faculty of Health and Life Sciences, Oxford Brookes University, Oxford, UK. ${ }^{7}$ These authors contributed equally: Harry M. Scholes, Adam Cryar, and Fiona Kerr. ${ }^{\boxplus}$ email: c.orengo@ucl.ac.uk; l.partridge@ucl.ac.uk; k.thalassinos@ucl.ac.uk
} 
and synaptic recycling pathways may also be required for $\mathrm{A} \beta 42$ to initiate the toxic cascade of events leading to tau pathology and neuronal damage in dementia.

Comparison of proteomic analyses of post-mortem human brains have further revealed an increase in metabolic processes and reduction in synaptic function in $\mathrm{AD}^{17}$. Oxidised proteins also accumulate at early stages in $\mathrm{AD}$ brain, probably as a result of mitochondrial ROS production ${ }^{18}$, and redox proteomic approaches suggest that enzymes involved in glucose metabolism are oxidised in mild cognitive impairment and $\mathrm{AD}^{19,20}$. Moreover, phospho-proteomic approaches have revealed alterations in phosphorylation of metabolic enzymes and kinases that regulate phosphorylation of chaperones such as HSP27 and crystallin alpha $\mathrm{B}^{21}$. Of note, however, there is little proteomic overlap between studies using post-mortem human brain tissue, which may reflect the low sample numbers available for such studies, differences in comorbidities between patients and confounding post-mortem procedures ${ }^{17}$. Although valuable, post-mortem studies also reflect the end-stage of disease and, therefore, do not facilitate measurement of dynamic alterations in proteins as AD progresses.

Animal models of AD, generated through transgenic over-expression of human APP or tau, provide an opportunity to track proteomic alterations at pre- and post-pathological stages, thus facilitating insight into the molecular mechanisms underlying disease development and revealing new targets for drugs to prevent AD progression. Analyses of transgenic mice models of AD have revealed some overlapping alterations in metabolic enzymes, kinases and chaperones with human $\mathrm{AD}$ brain ${ }^{17}$. Only one study, however, has tracked alterations in protein carbonylation over time, showing increases in oxidation of metabolic enzymes (alpha-enolase, ATP synthase $\alpha$-chain and pyruvate dehydrogenase E1) and regulatory molecules (14-3-3 and Pin1) in correlation with disease progression ${ }^{22}$.

Adult-onset Drosophila models of AD have been generated by over-expressing human A $\beta 42$ peptide exclusively in adult fly neurons using inducible expression systems. These models have been shown to develop progressive neurodegenerative phenotypes, such as reduced climbing ability, and shortened lifespan ${ }^{23}$. Taking advantage of the short lifespan of the fly, and the flexible nature of the inducible model, we have performed a longitudinal study of the brain proteome to capture the effects of A $\beta 42$-toxicity in the brain from the point of induction and across life. We identified 3093 proteins using label-free quantitative ion-mobility data independent analysis mass spectrometry (IM-DIA-MS) ${ }^{24}, 1854$ of which were common to healthy and A $\beta 42$ flies. Of these, we identified 228 proteins that were significantly altered in $\mathrm{AD}$, some of which overlapped with normal ageing but the majority of which were ageing-independent. Proteins altered in response to A $\beta 42$ were enriched for AD processes and have statistically significant network properties in the brain protein interaction network. We also show that these proteins are likely to be bottlenecks for signalling in the network, suggesting that they comprise important proteins for normal brain function. Our data is a valuable resource to begin to understand the dynamic properties of $A \beta 42$ proteo-toxicity during $A D$ progression. Future functional studies will be required to determine the causal role of these proteins in mediating progression of $\mathrm{AD}$ using model organisms and to translate these findings to mammalian systems.

\section{Results}

Proteome analysis of healthy and A $\beta 42$-expressing fly brains. Using an inducible transgenic fly line expressing human Arctic mutant $A \beta 42(\mathrm{TgAD})^{23}$ (Fig. 1a), we confirmed a previously observed ${ }^{23}$ reduction in lifespan following $A \beta 42$ induction prior to proteomic analyses (Fig. 1b).

To understand how the brain proteome is affected as $\mathrm{A} \beta 42$ toxicity progresses, fly brains were dissected from healthy and $A \beta 42$ flies at 5, 19, 31 and 46 days, and at 54 and 80 days for healthy controls, then analysed by labelfree quantitative IM-DIA-MS (Fig. 1c, Supplementary Data 1). 1854 proteins were identified in both healthy and $A \beta 42$ fly brain from a total of 3093 proteins (Fig. 1d), which is typical for recent fly proteomics studies ${ }^{25,26}$.

For the 1854 proteins identified in both healthy and A 342 flies, we assessed the reliability of our data. Proteins were highly correlated between technical and biological repeats (Fig. S1). We used principal component analysis of the protein abundances to identify sources of variance (Fig. 1e). Healthy and $A \beta 42$ samples are clearly separated in the first principal component, probably due to the effects of $A \beta 42$. In the second principal component, samples are separated by increasing age, due to age-dependent or disease progression changes in the proteome. These results show that whilst ageing does contribute to changes in the brain proteome $(8.7 \%$ of the total variance), much larger changes are due to expression of A $342(70.6 \%)$ and this may reflect either a correlation with the ageing process or progression of $\mathrm{AD}$ pathology. We confirmed this result using hierarchical biclustering of protein abundances in $A \beta 42$ versus healthy flies at 5 days (Fig. 1f). The results reveal that most proteins do not vary significantly in abundance with age in healthy flies, but many proteins are differentially abundant in $\mathrm{A} \beta 42$ flies.

Analysis of brain proteome dysregulation in $\mathbf{A} \beta \mathbf{4 2}$ flies. We next identified the proteins that were significantly altered following $A \beta 42$ expression in the fly brain. To achieve this, we used five methods commonly used to analyse time course RNA-Seq data ${ }^{27}$ and classified proteins as significantly altered if at least two methods detected them ${ }^{28}$. We identified 228 significantly altered proteins from 740 proteins that were detected by one or more methods (Fig. 2a; Supplementary Data 2). A comparison of popular RNA-Seq analysis tools ${ }^{29}$ showed that edge ${ }^{30}$ has a high false positive rate and variable performance on different data sets, whereas, DESeq2 ${ }^{31}$ and limma $a^{32}$ have low false positive rates and perform more consistently. We observed a similar trend in our data set. limma and DESeq2 detected the lowest number of proteins, with 21 proteins in common (Fig. S2a). edgeR detected more proteins, of which 38 were also detected by DESeq 2 and 16 by limma. EDGE ${ }^{33}$ and maSigPro ${ }^{34}$ detected vastly more proteins, 464 of which were only detected by one method. Principal component analysis shows that edgeR, DESeq2 and limma detect similar proteins, whereas, EDGE and maSigPro detect very different proteins (Fig. S2b). 
a

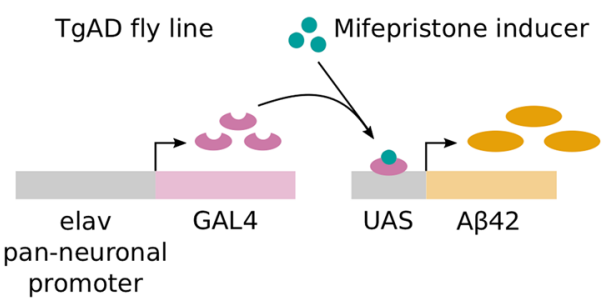

C

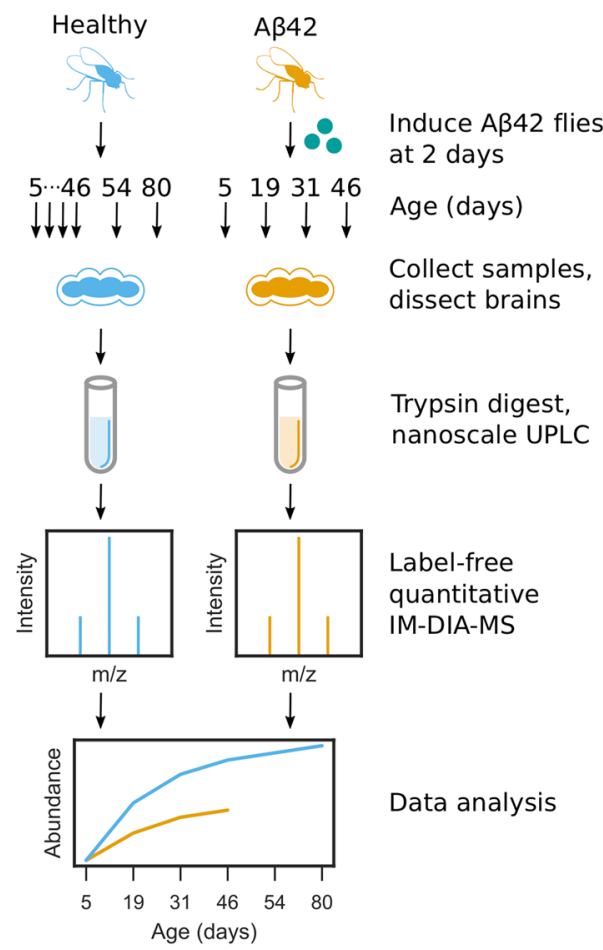

f

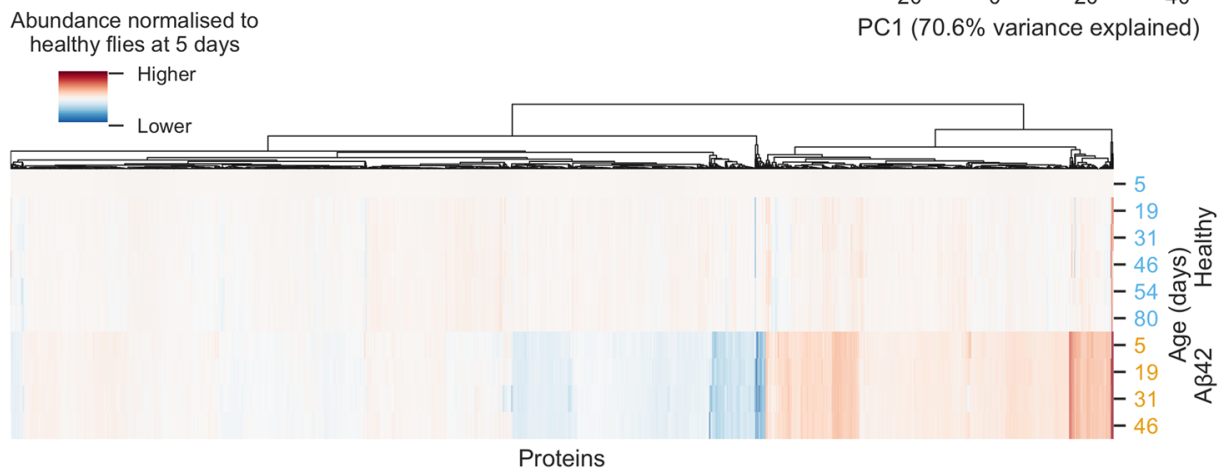

Figure 1. Proteome analysis of healthy and AD fly brains. (a) Drosophila melanogaster transgenic model of $\mathrm{AD}(\mathrm{TgAD})$ that expresses Arctic mutant $\mathrm{A} \beta 42$ in a mifepristone-inducible GAL4/UAS expression system under the pan-neuronal elav promoter. (b) Survival curves for healthy and A $\beta 42$ flies. A $\beta 42$ flies were induced to express $A \beta 42$ at 2 days. Markers indicate days that MS samples were collected. (c) Experimental design of the brain proteome analysis. A $\beta 42$ flies were induced to express $A \beta 42$ at 2 days. For each of the three biological repeats, 10 healthy and $10 \mathrm{~A} \beta 42$ flies were collected at 5, 19, 31 and 46 days, as well as 54 and 80 days for healthy flies. Proteins were extracted from dissected brains and digested with trypsin. The resulting peptides were separated by nanoscale liquid chromatography and analysed by label-free quantitative IM-DIA-MS. (d) Proteins identified by IM-DIA-MS. (e) Principal component analysis of the IM-DIA-MS data. Axes are annotated with the percentage of variance explained by each principal component. (f) Hierarchical biclustering using relative protein abundances normalised to their abundance in healthy flies at 5 days. 
a

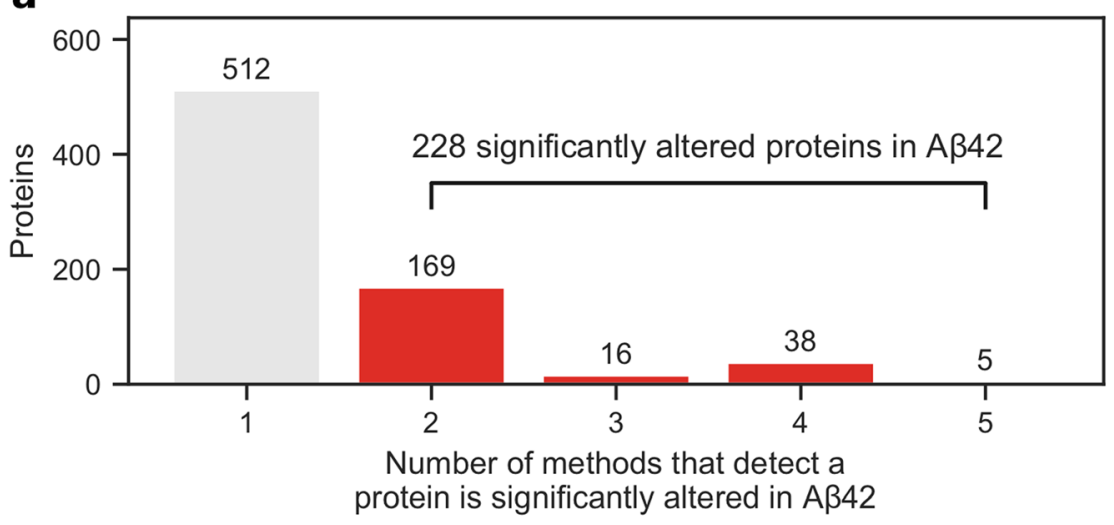

b

Significantly altered proteins

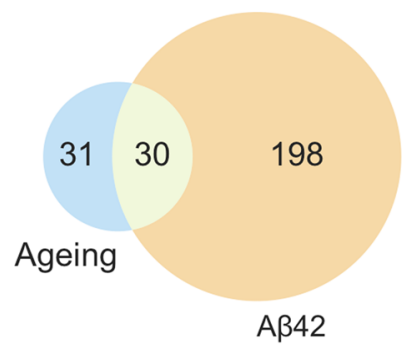

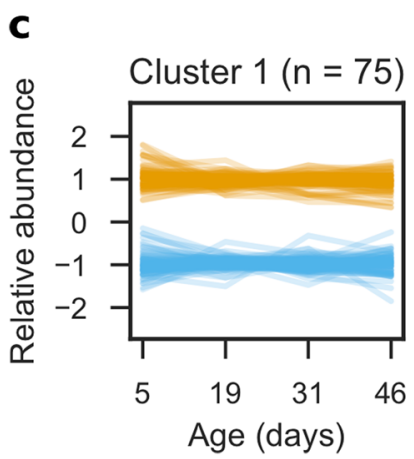
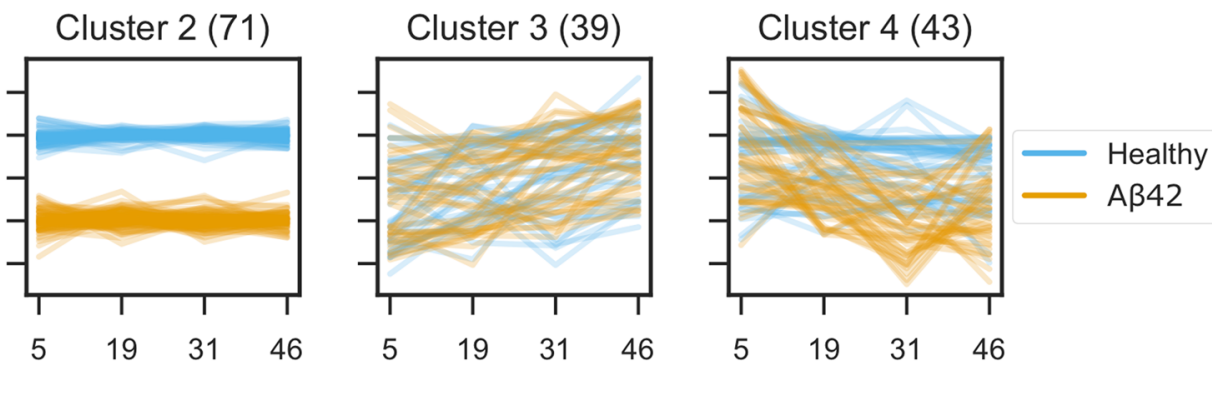

Figure 2. Brain proteome dysregulation in AD. (a) Proteins significantly altered in AD were identified using five methods (EDGE, edgeR, DESeq2, limma and maSigPro) and classified as significantly altered if at least two methods detected them. (b) Significantly altered proteins in AD (from a) and ageing. (c) Significantly altered protein abundances were $z$ score-transformed and clustered using a Gaussian mixture model.

Although these methods should be able to differentiate between proteins that are altered in A $\beta 42$ flies from those that change during normal ageing, we confirmed this by analysing healthy flies separately. In total, 61 proteins were identified as significantly altered with age (Fig. S3; Supplementary Data 3), of which 30 were also identified as significantly altered in $\mathrm{AD}$ (Fig. 2b) and 31 in normal ageing alone. These proteins are not significantly enriched for any pathways or functions. Based on our results, we concluded that the vast majority of proteins that are significantly altered in $\mathrm{AD}$ are not altered in normal ageing and that $\mathrm{AD}$ causes significant dysregulation of the brain proteome.

Of the 31 proteins specifically altered in ageing (Fig. S4), 10 decreased with age (Acp1, CG7203, mRPL12, qm, CG11017, HIP, HIP-R, PP02 and Rpn1), 15 increased with age (ade5, CG7352, RhoGAP68F, CG9112, PCB, Aldh, D2hgdh, CG7470, CG7920, RhoGDI, Aldh7A1, CG8036, Ssadh, muc and FKBP14) and four fluctuated throughout life (CG14095, His2A, RpL6 and SERCA).

To understand the dynamics of protein alterations following $A \beta 42$ induction, we clustered the profiles of proteins significantly altered in A $\beta 42$ flies using a Gaussian mixture model (Fig. $2 \mathrm{c}$ ). The proteins clustered best into four sets (Fig. S5), determined by the BIC, which was smallest with four clusters. In comparison to healthy flies, cluster 1 contains proteins that have consistently higher abundance in $\mathrm{A} \beta 42$ flies. Conversely, cluster 2 contains proteins that have lower abundance in A $\beta 42$ flies. The abundances of proteins from clusters 1 and 2 are affected from the onset of disease at day 5 , and remain at similar levels as the disease progresses. Proteins in cluster 3 follow a similar trend in healthy and $\mathrm{A} \beta 42$ flies and increase in abundance with age. However, cluster 4 proteins decrease in abundance as the disease progresses, whilst remaining steady in healthy flies.

We performed a statistical Gene Ontology enrichment analysis on each cluster but found no enrichment of terms. Furthermore, we also saw no enrichment when we analysed all 228 proteins together.

Brain proteins significantly altered by $\mathrm{A} \beta 42$ have distinct network properties. Following the analyses of brain proteome dysregulation in A $\beta 42$ flies, we analysed the 228 significantly altered proteins in the context of the brain protein interaction network to determine whether their network properties are significantly different to the other brain proteins. We used a subgraph of the STRING ${ }^{35}$ network induced on the 3093 proteins identified by IM-DIA-MS (see Networks section in the Methods for more details). This subgraph contained 183 of the 228 significantly altered proteins (Fig. S6; Supplementary Data 4). We then calculated four graph theoretic network properties (Fig. 3a) of these 183 significantly altered proteins contained in this network: degree, the number of edges that a node has; shortest path, the smallest node set that connect any two nodes; largest connected component, the largest node set for which all nodes have at least one edge to any of the other nodes; and betweenness centrality, the proportion of all shortest paths in the network that a particular node lies on. 


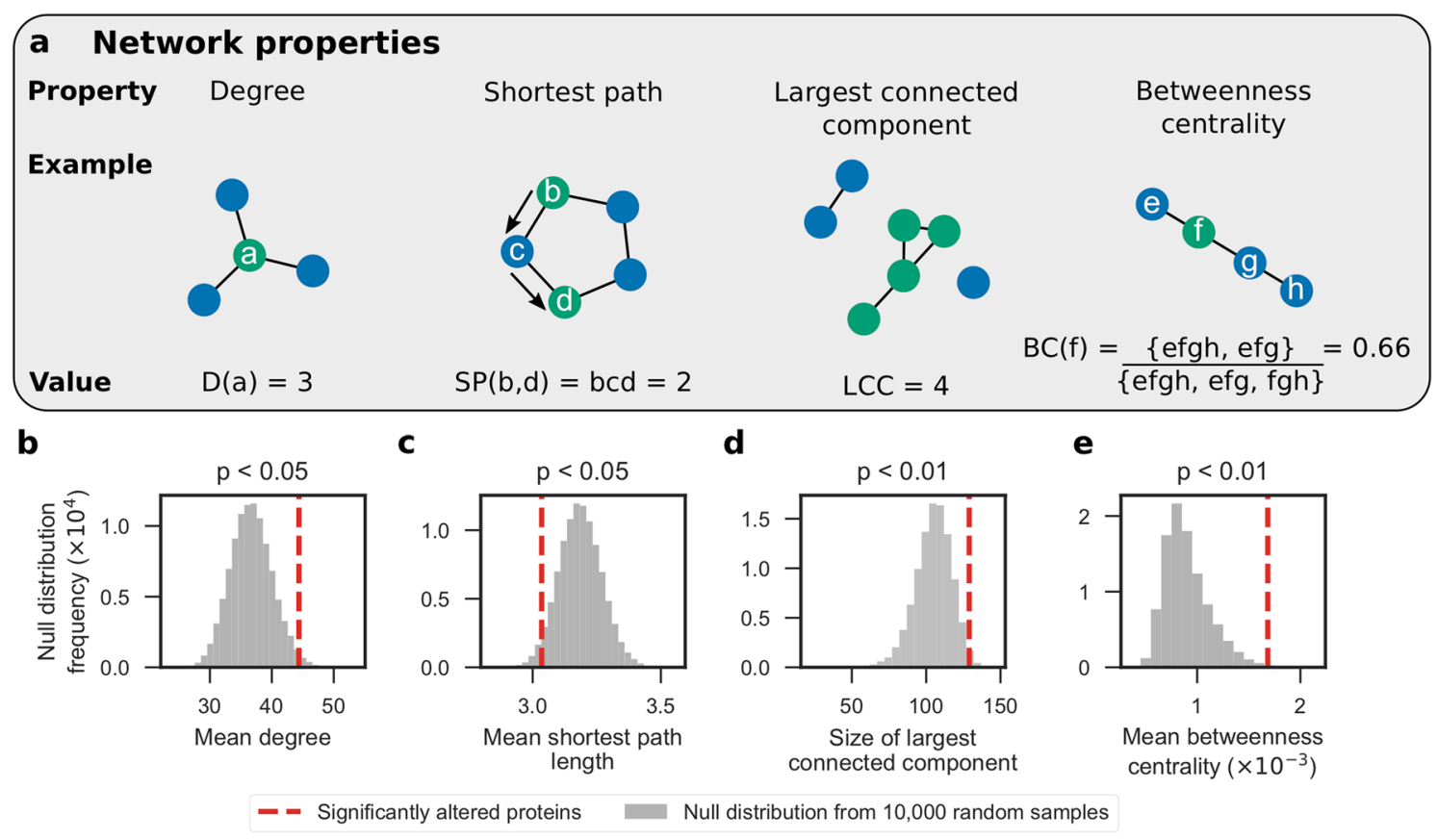

Figure 3. Significantly altered proteins have statistically significant network properties in the brain protein interaction network. (a) Network properties that were calculated: degree, the number of edges that a node has; shortest path, the smallest node set that connect any two nodes; largest connected component, the largest node set for which all nodes have at least one edge to any of the other nodes; and betweenness centrality, the proportion of all shortest paths in the network that a particular node lies on. Using a subgraph of the STRING network induced on the 3093 proteins identified by IM-DIA-MS in healthy and A 342 flies, the significance of four network characteristics were calculated for the 183 significantly altered proteins contained in this subgraph. (b) Mean degree; (c) mean shortest path length between a node and the remaining 182 nodes; (d) the size of the largest connected component in the subgraph induced on these nodes; and (e) mean betweenness centrality. One-sided non-parametric $P$ values were calculated using null distributions of the test statistics, simulated by randomly sampling 183 nodes from the network 10,000 times.

We performed hypothesis tests and found that these proteins have statistically significant network properties. Firstly, the significantly altered proteins make more interactions than expected (mean degree $P<0.05$; Fig. $3 \mathrm{~b}$ ). Therefore, these proteins may further imbalance the proteome by disrupting the expression or activity of proteins they interact with. Secondly, not only are these proteins close to each other (mean shortest path $P<0.05$; Fig. 3c), but also 129 of them form a connected component (size of largest connected component $P<0.01$; Fig. 3d). These two pieces of evidence suggest that $\mathrm{A} \beta 42$ disrupts proteins at the centre of the proteome. Lastly, these proteins lie along shortest paths between many pairs of nodes (mean betweenness centrality $P<0.01$; Fig. $3 \mathrm{e}$ ) and may control how signals are transmitted in cells. Proteins with high betweenness centrality are also more likely to be essential genes for viability ${ }^{36}$. Taken together, these findings suggest that the proteins significantly altered in AD are important in the protein interaction network.

Predicting the severity of $A \beta 42$-induced protein alterations using network properties. We predicted how severely particular A $\beta 42$-associated protein alterations may affect the brain using two network properties-the tendency of a node to be a hub or a bottleneck. In networks, nodes with high degree are hubs for communication, whereas nodes with high betweenness centrality are bottlenecks that regulate how signals propagate through the network. Protein expression tends to be highly correlated to that of its neighbours in the protein interaction network. One exception to this rule, however, are bottleneck proteins, whose expression tends to be poorly correlated with that of its neighbours ${ }^{36}$. This suggests that the proteome is finely balanced and that the expression of bottleneck proteins is tightly regulated to maintain homeostasis. We analysed the hub and bottleneck properties of the significantly altered proteins and identified four hub-bottlenecks and five nonhubbottlenecks that correlate with $\mathrm{A} \beta 42$ expression (Fig. 4a) and analysed how their abundances change during normal ageing and as pathology progresses (Fig. $4 \mathrm{~b}$ ).

Non-hub bottleneck proteins (Fig. 4b) Acs1 and Got2 levels were stably expressed throughout normal ageing in our healthy flies but increased upon $A \beta 42$ induction and continued to rise with age in $A \beta 42$ flies. On the other hand, Echs1 abundance increased in healthy flies during normal ageing, but its levels were reduced upon A $\beta 42$ induction and its ageing-dependent increase was diminished in A $\beta 42$ flies compared to controls. Levels of $\mathrm{mt}$ :CoII (a COX subunit) declined with age in healthy control, but not in $A \beta 42$, fly brain, although its expression was downregulated compared to controls at all time-points following A $\beta 42$ induction. Finally, the cuticle protein Acp65Aa was also upregulated in A $\beta 42$ flies compared to controls, but levels fell sharply between 5 and 19 days of age. 
a

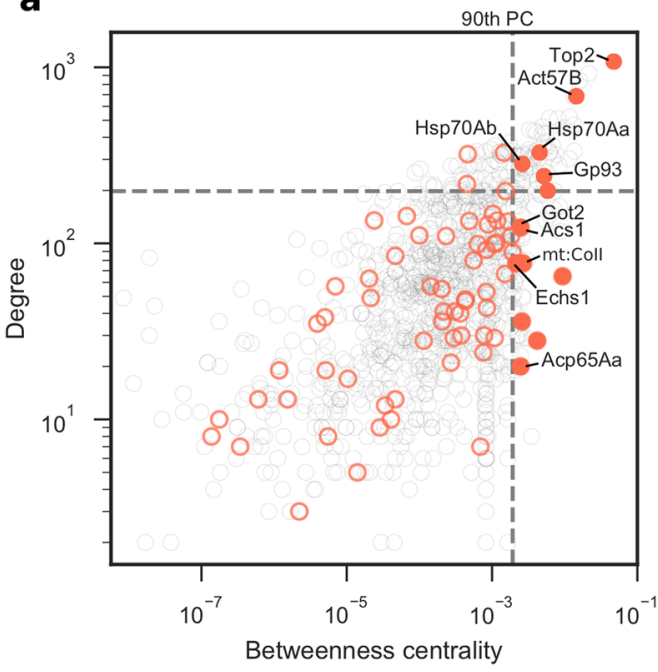

b

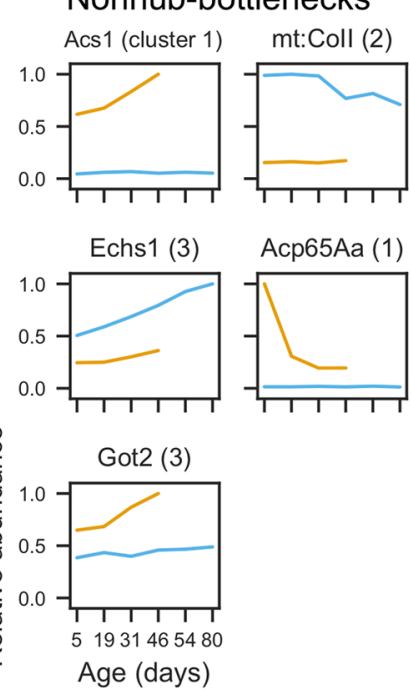

Hub-bottlenecks
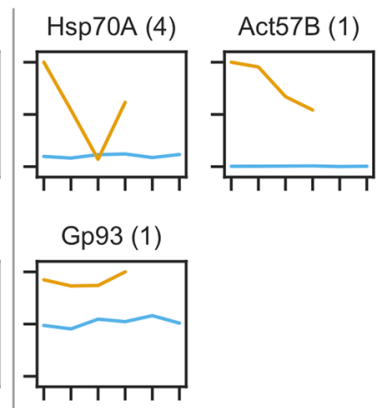

Top2 (4)

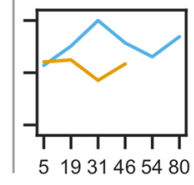

Figure 4. Analysis of hubs and bottlenecks in the brain protein interaction network. In networks, nodes with high degree are hubs and nodes with high betweenness centrality are bottlenecks. (a) Degree (hub-ness) is plotted against betweenness centrality (bottleneck-ness) in the brain protein interaction network for all proteins identified by IM-DIA-MS (grey circles). Of the significantly altered proteins (red circles), hub-bottleneck (>90th percentile (PC) for degree and betweenness centrality) and nonhub-bottleneck proteins (>90th PC for betweenness centrality) are highlighted (filled red circles). (b) Profiles of significantly altered bottleneck proteins implicated in A $\beta 42$ toxicity. Maximum abundances are scaled to 1. Numbers in parentheses denote which cluster from Fig. $2 \mathrm{c}$ the protein was in.

Of the four hub-bottlenecks (Fig. 4b) Hsp70A was significantly upregulated at early time-points (5 days) in $A \beta 42$ flies, dropped between days 5 and 31 post-induction, then increased at later time-points, compared to healthy controls which exhibited stable expression of this protein throughout life. We found that Gp93 was increased across age in A $\beta 42$ flies compared to controls, possibly suggesting an early and sustained protective mechanism against A $\beta 42$-induced damage. DNA topoisomerase 2 (Top2), an essential enzyme for DNA doublestrand break repair, was decreased in A $\beta 42$ flies, following a pattern which mirrors changes in its expression with normal ageing. Finally, we found that actin (Act57B) was increased in A $\beta 42$ flies but declined with age, in comparison to control fly brains which displayed stable expression across life.

Due to the importance of these hub and bottleneck proteins in the protein interaction network, we predict that $\mathrm{AD}$-associated alterations in their abundance will likely have a significant effect on the cellular dynamics of the brain.

Dysregulated genes are associated with known AD and ageing network modules. Finally, we clustered the protein interaction network into modules and performed a Gene Ontology enrichment analysis on modules that contained any of the 228 significantly altered proteins. We saw no Gene Ontology term enrichment when we tested these proteins clustered according to their abundance profiles (Fig. 2c), presumably because the proteins affected in $\mathrm{AD}$ are diverse and involved in many different biological processes. However, by testing network modules for functional enrichment, we exploited the principle that interacting proteins are functionally associated. Using a subgraph of the STRING network containing the significantly altered proteins and their directly-interacting neighbours (see Networks section in the Methods for more details), we used MCODE ${ }^{37}$ to find modules of densely interconnected nodes. We chose to include neighbouring proteins to compensate for proteins that may not have been detected in the MS experiments due to the stochastic nature of observing peptides and the wide dynamic range of biological samples ${ }^{38}$. The resulting subgraph contained 4842 proteins, including 183 of the 228 significantly altered proteins, as well as 477 proteins that were only identified in healthy or A $\beta 42$ flies and 3125 proteins that were not identified in our IM-DIA-MS experiments. 12 modules were present in the network (Fig. 5, Supplementary Data 5). Module sizes range from 302 to 17 nodes. The smallest module (MCODE module 8) is shown in Fig. S7. The proportion of these modules that were composed of significantly altered proteins ranged from 0 to $8 \%$. All but one of the modules were enriched for processes implicated in $\mathrm{AD}$ and ageing (Fig. 5, Supplementary Data 6), including respiration and oxidative phosphorylation, transcription and translation, proteolysis, DNA replication and repair, and cell cycle regulation. ApoB was found in the second highest scoring module that contains proteins involved in translation and glucose transport55 (Fig. 5).

We analysed the 31 proteins significantly altered in normal ageing, but not AD (Supplementary Data 3). Of the 29 proteins that were contained in the STRING network, 24 interact directly with at least one of the AD significantly altered proteins, suggesting an interplay between ageing and $\mathrm{AD}$ at the pathway level. Using a subgraph of the STRING network induced on these proteins and their 1603 neighbours, we identified eight network modules (Supplementary Data 7) that were enriched for ageing processes ${ }^{39}$, including respiration, unfolded protein and oxidative damage stress responses, cell cycle regulation, DNA damage repair, and apoptosis. 


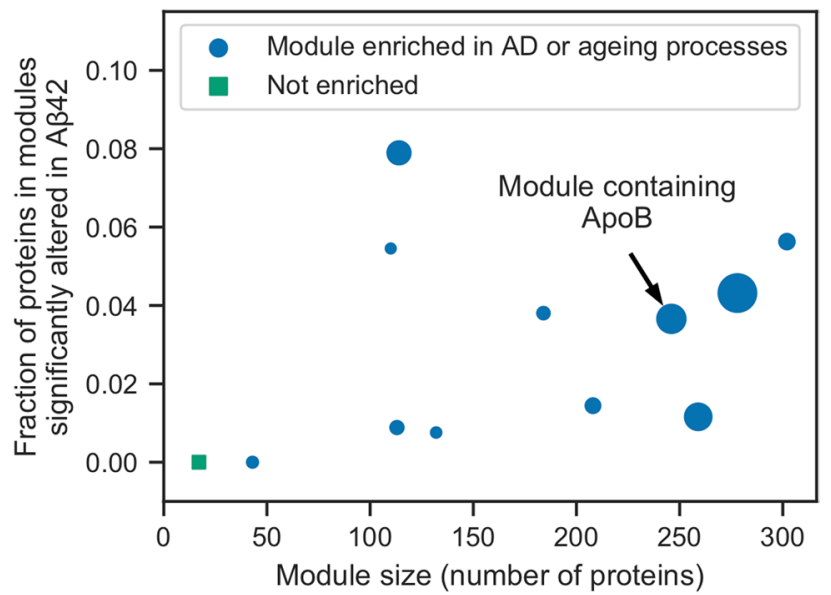

Figure 5. Analysis of network modules enriched for AD or ageing processes. MCODE was used to identify network modules in a subgraph of the STRING network containing the significantly altered proteins and their directly-interacting neighbours. The size of the resulting 12 modules is plotted against the fraction of proteins in these modules that are significantly altered in AD. Module 2 is annotated as containing ApoB. Marker sizes denote the MCODE score for the module.

\section{Discussion}

Despite the substantial research effort spent on finding drugs against $\mathrm{AD}$, effective treatments remain elusive. There is a need to better understand the molecular processes that govern the onset and progression of the complex pathologies observed in this condition to help identify new drug targets to treat and prevent AD.

Analysis of post-mortem human brain tissue is an important way to study dementia, but cannot capture the progression of pathology from the initiation of disease. Due to their short lifespan and ease of genetic manipulation, model organisms such as Drosophila melanogaster provide a tractable system in which to examine the progression of AD pathology across life. We performed a longitudinal study of the Drosophila brain proteome, using an inducible model of $\mathrm{AD}$, label-free quantitative IM-DIA-MS and network analyses. We were able to track alterations in protein levels from the point of exposure to human $A \beta 42$ and the widespread interaction of $A \beta 42$ with brain signalling networks as pathology progresses over the life course.

We identified 61 proteins that were significantly altered with age in fly brain, 31 of which were not altered in response to $A \beta 42$. Of these, structural chitin proteins (Acp1 and CG7203), mitochondrial associated proteins (HemK1 and mRPL12), geranylgeranyl transferases (qm), and proteostasis proteins (HIP, HIP-R and Rpn1) were significantly downregulated with age. In agreement with these observations, loss of mitochondrial function and proteostasis are key features of the ageing brain ${ }^{40}$. Recent studies also suggest a role for geranylgeranyl transferase I-mediated protein prenylation in mediating synaptogenesis and learning and memory ${ }^{41,42 .}$ Our findings suggest that this may represent a novel mechanism of regulation and maintenance of these functions during ageing, which warrants further investigation. Proteins involved in inositol monophosphate (IMP) biosynthesis (ade5), endosome recycling (RhoGAP68F and RhoGDI), oxidation-reduction processes (D2hgdh, Aldh and Aldh7A1), pyruvate metabolism (PCB and muc), neurotransmitter function (Ssadh), and ER-related protein folding (FKBP14) were increased with age in our study. Oxidative damage is a key feature of ageing brain and loss of aldehyde dehydrogenase (Aldh) function, which detoxifies oxidative stress inducing aldehydes, has been shown to be associated with promoting age-related neurodegeneration and cognitive dysfunction in mice ${ }^{43,44}$. Phosphatidylinositol signalling is important for stabilising mood and behaviour, and IMP inhibition is thought to partially mediate the beneficial effects of lithium in the treatment of bipolar disorder ${ }^{45}$. Rho GTPases are involved in maintenance of synaptic function ${ }^{41}$, and reductions in their levels correlate with ageing and increases in their expression with foraging behaviour in the brain of honey bees ${ }^{46}$. Our finding that inhibitors of these enzymes (Rho GAPs and Rho GDIs) are upregulated in ageing fly brain further suggests that changes in their activity may mediate loss of synaptic function throughout life. Ageing is also characterised by a progressive loss of metabolic function, and studies suggest that ATP-generating metabolites, such as pyruvate, improve cognitive function ${ }^{47}$ as reflected by upregulation of pyruvate carboxylase and muc, a component of the pyruvate dehydrogenase complex, in our data set. Finally, several proteins fluctuated in expression across age in our flies, including those involved in DNA repair (His2A), protein translation (RpL6), and ER calcium homeostasis (SERCA), processes which have been previously reported in association with brain ageing ${ }^{48-50}$. Although alterations in these proteins are independent of $A \beta 42$ expression in our flies, further work is required to investigate their functional role in preserving brain function with age and their potential to increase the vulnerability of the ageing brain to neurodegenerative diseases.

Our proteomic analyses identified A 342 -induced alterations in levels of 228 proteins, which clustered into four groups. First, those which were either elevated (cluster 1) or reduced (cluster 2) in AD relative to controls throughout life, and dysregulation of which may initiate AD pathogenesis, may be involved in early stages of disease progression, or represent defense mechanisms that could be harnessed for protection. Second, those 
which were altered in correlation with ageing in healthy and $A \beta 42$ flies (cluster 3). Finally, those which changed in A $\beta 42$ flies across life but independently of ageing-dependent effects in healthy controls (cluster 4). Further work is required to determine whether reduction of these proteins plays a causal role in disease pathogenesis that could be targeted therapeutically, or whether their decline represents a protective response to damage.

Moreover, computational analysis of these proteins revealed significant network properties within the fly brain proteome. Assessing hub and bottleneck properties, many of the A 342 -induced proteomic changes represented alterations in bottleneck proteins suggesting that they play key roles in downstream cellular function. Of these, some display non-hub properties indicating that they are important for maintaining cellular homeostasis in a targeted fashion, whereas others also displayed hub properties suggesting that they are central in linking cellular signalling pathways to maintain cell function.

During the review of this manuscript, we were made aware of a study that compares the performance of different normalisation methods on quantitative label-free proteomics data ${ }^{51}$. The authors of this study found that the variance stabilisation normalisation has the most desirable characteristics for normalising data from quantitative label-free proteomics. In this work, we used a quantile normalisation, however, we do not believe this method introduced any undesirable characteristics or biases.

We identified five nonhub-bottleneck proteins and four hub-bottleneck proteins, the expression of which was altered in $\mathrm{A} \beta 42$ flies relative to controls across life. Due to the importance of these hub and bottleneck proteins in the protein interaction network, we predict that $\mathrm{AD}$-associated alterations in their abundance will likely have a significant effect on the cellular dynamics of the brain. Indeed, some of these proteins play key molecular roles in metabolism, protein homeostasis, protection against oxidative stress, and DNA damage, processes which have been shown to affect neuronal function and protect against proteo-toxicity. Acyl-CoA synthetase long chain (Acs1), Enoyl-CoA hydratase, short chain 1 (Echs1), and Aspartate aminotransferase (Got2), are metabolic enzymes with previous links to neuronal function and damage ${ }^{6}$. Got2 produces the neurotransmitter L-glutamate from aspartate, is involved in assembly of synapses and becomes elevated following brain injury ${ }^{52}$. Cytochrome $\mathrm{c}$ oxidase (COX), complex IV of the mitochondrial electron transport chain, uses energy from reducing molecular oxygen to water to generate a proton gradient across the inner mitochondrial membrane. Although $A \beta$ is known to inhibit COX activity ${ }^{53}$, the link between COX and AD is unclear. In AD patients, COX activity-but not abundance-is reduced, resulting in increased levels of ROS ${ }^{54}$. However, in COX-deficient mouse models of $\mathrm{AD}$, plaque deposition and oxidative damage are reduced ${ }^{55}$. Hsp70A is a heat shock protein that responds to hypoxia and Gp93 is a stress response protein that binds unfolded proteins, consistent with responses to abnormal A 342 aggregation in our flies. DNA topoisomerase 2 (Top2) is an essential enzyme for DNA double-strand break repair. Double-strand breaks occur naturally in the brain as a consequence of neuronal activity-an effect that is aggravated by $A \beta^{7}$. As a consequence of deficient DNA repair machinery, deleterious genetic lesions may accumulate in the brain and exacerbate neuronal loss. The cuticle protein Acp65Aa is a chitin, this class of which have been detected in $\mathrm{AD}$ brains and suggested to facilitate $\mathrm{A} \beta$ nucleation ${ }^{56}$. Finally, actin (Act57B) is a structural protein, and depolymerisation of F-actin filaments have been observed in a mouse AD model before onset of $\mathrm{AD}$ pathology $\mathrm{y}^{57}$. Alterations in these proteins may represent either adaptive responses to the presence of abnormal protein aggregates, such as $\mathrm{A} \beta 42$, or mediators of neuronal toxicity. Further functional genomic studies are therefore required to establish the causal role of these processes in governing onset and progression of AD pathology.

Assessing the human orthologs of these genes, identified using DIOPT ${ }^{58}$, indicates that several of these bottleneck proteins have been previously implicated in association with $\mathrm{AD}$ or other neurological conditions in humans or mammalian models of disease. ACSL4 (Acs1 ortholog) has been shown to associate with synaptic growth cone development and mental retardation ${ }^{59}$. Mutations in ECHS1 (Echs1 ortholog), an enzyme involved in mitochondrial fatty acid oxidation, associate with Leigh Syndrome, a severe developmental neurological disorder ${ }^{60}$. Proteomic studies have revealed that GOT2 (Got2 ortholog) is down-regulated in infarct regions following stroke $^{61}$, and in AD patient brain ${ }^{62}$. Integrating data from human post-mortem brain studies, HSPA1A (Hsp70Aa ortholog) upregulates in the protein interaction network of AD patients compared to healthy controls ${ }^{63}$, and has recently been suggested to block APP processing and A $\beta$ production in mouse brain ${ }^{64}$. Synthetic, fibrillar, A $\beta 42$ reduces expression of TOP2B (Top2 ortholog) in rat cerebellar granule cells and in a human mesenchymal cell line, suggesting this may contribute to DNA damage in response to amyloid ${ }^{65}$. HSP90B1 (Gp93 ortholog) shows increased expression following TBI in mice ${ }^{66}$, and associates with animal models of Huntington's disease ${ }^{67}$. Finally, ACTB (Act57B ortholog) has been implicated as a significant AD risk gene and central hub node using integrated network analyses across GWAS ${ }^{68}$.

ACSL4, ECHS1, and HSP90B1 have no reported association with AD or related dementias, however, which suggests that our study has the potential to identify new targets in the molecular pathogenesis of this disease. Our study also provides additional information about the homeostasis of these proteins across life from the point of amyloid production. For example, the abundances of Acs 1 and Got2 are elevated following A $\beta 42$ induction and continue to increase with age relative to controls. Echs 1 is reduced in A $\beta 42$ flies compared to controls but increases across life in parallel with ageing-dependent increases in this protein. Structural proteins Acp65Aa and $A c t 57 \mathrm{~B}$ are elevated in response to $\mathrm{A} \beta 42$ but decline across life whilst remaining stable in control flies. Gp93 and Top2 are either elevated or reduced in response to $\mathrm{A} \beta 42$ but mirror ageing-dependent alterations in their expression. $\mathrm{mt}$ :CoII is reduced following $\mathrm{A} \beta 42$ expression at all time-points, but reduced with ageing in controls. Hsp70A is increased early in A $\beta 42$ flies, reduced to control levels in mid-life then elevated at late pathological stages whilst remaining stable in healthy controls.

Analysing Gene Ontology enrichment using network modules, to capture the diverse biological processes modified in $\mathrm{AD}$, we identified 12 modules enriched for processes previously implicated in ageing and $\mathrm{AD}$. This validates the use of our Drosophila model in identifying progressive molecular changes in response to A $\beta 42$ that are likely to correlate with progression of cognitive decline in human disease. Further work is required to modify 
the genes identified in our study at different ages, in order to elucidate whether they represent mediators of toxicity as the disease progresses, factors which increase neuronal susceptibility to disease with age or compensatory protective mechanisms. Model organisms will be essential in unravelling these complex interactions. Our study therefore forms a basis for future analyses that may identify new targets for disease intervention that are specific to age and/or pathological stage of AD.

\section{Materials and methods}

Fly stocks. The TgAD fly line used in this study ${ }^{23}$ contains the human transgene encoding the Arctic mutant A $\beta 42$ peptide under the control of an Upstream Activation Sequence (UAS) ${ }^{69}$. Expression of A $\beta 42$ was controlled by GeneSwitch ${ }^{70}$ - a mifepristone-inducible GAL4/UAS expression system — under the pan-neuronal elav promoter. All flies were backcrossed for six generations into the $\mathrm{w}^{1118}$ genetic background.

Flies were grown in $200 \mathrm{ml}$ bottles on a $12 \mathrm{~h} / 12 \mathrm{~h} \mathrm{light} /$ dark cycle at constant temperature $\left(25^{\circ} \mathrm{C}\right)$ and humidity. Growth media contained $15 \mathrm{~g} / \mathrm{l}$ agar, $50 \mathrm{~g} / \mathrm{l}$ sugar, $100 \mathrm{~g} / \mathrm{l}$ autolysed yeast, $100 \mathrm{~g} / \mathrm{l}$ nipagin and $3 \mathrm{ml} / \mathrm{l}$ propionic acid. Flies were maintained for two days after eclosion before females were transferred to vials at a density of 25 flies per vial for the lifespan analysis and 10 flies per vial for the IM-DIA-MS analysis. Expression of A $\beta 42$ was induced in TgAD flies by spiking the growth media with mifepristone to a final concentration of $200 \mu \mathrm{M}$. Flies were transferred to fresh media three times per week, at which point the number of surviving flies was recorded. For each of the three biological repeats, 10 healthy and $10 \mathrm{~A} \beta 42$ flies were collected at 5, 19, 31 and 46 days, as well as 54 and 80 days for healthy flies. Following anesthetisation with $\mathrm{CO}_{2}$, brains were dissected in ice cold $10 \mathrm{mM}$ phosphate buffered saline snap frozen and stored at $-80^{\circ} \mathrm{C}$.

Extraction of brain proteins. Brain proteins were extracted by homogenisation on ice into $50 \mu \mathrm{l}$ of $50 \mathrm{mM}$ ammonium bicarbonate, $10 \mathrm{mM}$ DTT and $0.25 \%$ RapiGest detergent. Proteins were solubilised and disulfide bonds were reduced by heating at $80^{\circ} \mathrm{C}$ for $20 \mathrm{~min}$. Free cysteine thiols were alkylated by adding $20 \mathrm{mM}$ IAA and incubating at room temperature for $20 \mathrm{~min}$ in darkness. Protein concentration was determined and samples were diluted to a final concentration of $0.1 \%$ RapiGest using $50 \mathrm{mM}$ ammonium bicarbonate. Proteins were digested with trypsin overnight at $37^{\circ} \mathrm{C}$ at a 50:1 protein:trypsin ratio. Additional trypsin was added at a 100:1 ratio the following morning and incubated for a further hour. Detergent was removed by incubating at $60{ }^{\circ} \mathrm{C}$ for $1 \mathrm{~h}$ in $0.1 \%$ formic acid. Insoluble debris was removed by centrifugation at $14,000 \times g$ for $30 \mathrm{~min}$. Supernatant was collected, lyophilised and stored at $-80^{\circ} \mathrm{C}$. Prior to lyophilisation peptide concentration was estimated by nanodrop (Thermo Fisher Scientific, Waltham, MA).

Label-free quantitative IM-DIA-MS. Peptides were separated by nanoscale liquid chromatography (LC) by loading $300 \mathrm{ng}$ of protein onto an analytical reversed phase column. IM-DIA-MS analysis was performed using a Synapt G2-Si mass spectrometer (Waters Corporation, Manchester, UK). The time-of-flight analyzer of the instrument was externally calibrated with a NaCsI mixture from m/z 50 to 1990 . Spectra were acquired over a range of 50-2000 m/z. Each biological repeat was analysed at least twice to account for technical variation.

LC-MS data were peak detected and aligned by Progenesis QI for proteomics (Waters Corporation). The principles of the embedded search algorithm for DIA data has been described previously ${ }^{71}$. Proteins were identified by searching against the Drosophila melanogaster proteome in UniProt, appended with common contaminants, and reversed sequence entries to estimate protein identification false discovery rate (FDR) values, using previously specified search criteria ${ }^{72}$. Peptide intensities were normalised to control for variation in protein loading and relative quantification. Abundances were estimated by Hi3-based quantitation ${ }^{73}$.

Data analysis. Proteins that were identified in both healthy and A $\beta 42$ flies were considered for further analysis. Missing data were replaced by the minimum abundance measured for any protein in the same repeat ${ }^{38}$. The data were quantile normalised ${ }^{74}$, so that different conditions and time points could be compared reliably. Quantile normalisation transforms the abundances so that each repeat has the same distribution.

For PCA analysis, the data were $\log _{10}$-transformed and each protein was standardised to zero mean and unit variance. Hierarchical biclustering was performed using the Euclidean distance metric with the complete linkage method. Prior to clustering, proteins were normalised to their abundance in healthy flies at 5 days.

Proteins that were identified by IM-DIA-MS in either healthy or A $\beta 42$ flies were assessed for overrepresentation of Gene Ontology terms using GOrilla ${ }^{75}$, which uses ranked lists of target and background genes. Proteins were ranked in descending order by their mean abundance. The type I error rate was controlled by correcting for multiple testing using the Benjamini-Hochberg method at an FDR of 5\%.

Clusters of proteins were assessed for overrepresentation of GO-Slim terms in the Biological Process ontology using Panther (version 13.1) with a custom background of the 3093 proteins identified by IM-DIA-MS in healthy or AD flies.

Identification of significantly altered proteins. Significantly altered proteins were identified using five methods that are frequently used to identify differentially expressed genes in time course RNA-Seq data. $\mathrm{DESeq}^{31}, \mathrm{EDGE}^{33}$, edgeR ${ }^{30}, \operatorname{limma}^{32}$ and $\mathrm{maSigPro}{ }^{34}$ are all available in $\mathrm{R}$ through Bioconductor. Dispersions were estimated from the biological and technical repeats. Unless otherwise stated, default parameters were used for all methods under the null hypothesis that a protein does not change in abundance between healthy and AD conditions in normal ageing. The type I error rate was controlled by correcting for multiple testing using the Benjamini-Hochberg method at a FDR of $5 \%$. A protein was classified as significantly altered if two or more methods identified it. 
DESeq2 models proteins with the negative binomial distribution and performs likelihood ratio tests. A time course experiment was selected in EDGE using the likelihood ratio test and a normal null distribution. edgeR uses the negative binomial distribution and performs quasi-likelihood tests. limma fits linear models to the proteins and performed empirical Bayes F-tests. maSigPro fits generalised linear models to the proteins and performs log-likelihood ratio tests.

Significantly altered proteins were clustered using a Gaussian mixture model. Protein abundances were $\log 10$ transformed and $z$ scores were calculated. Gaussian mixture models were implemented for 1-228 clusters. The best model was chosen using the Bayesian information criterion (BIC), which penalises complex models:

$$
\mathrm{BIC}=-2 \ln (\mathrm{L})+\ln (\mathrm{n}) \mathrm{k}
$$

where $\ln (\mathrm{L})$ is the log-likelihood of the model, $\mathrm{n}$ is the number of significantly altered proteins and $\mathrm{k}$ is the number of clusters. The model with lowest BIC was chosen.

Networks. All network analysis was performed using the Drosophila melanogaster STRING network (version 10$)^{35}$. Low confidence interactions with a 'combined score' $<0.5$ were removed in all network analyses.

Network properties of the significantly altered proteins were analysed in the brain protein interaction network. A subgraph of the STRING network was induced on the 3093 proteins identified by IM-DIA-MS in healthy or A 342 flies and the largest connected component was selected (2428 nodes and 44,561 edges). The subgraph contained 183 of the 228 significantly altered proteins (Fig. S6; Supplementary Data 4). For these proteins, four network properties were calculated as test statistics: mean node degree; mean unweighted shortest path length between a node and the remaining 182 nodes; the size of the largest connected component in the subgraph induced on these nodes; and mean betweenness centrality. Hypothesis testing was performed using the null hypothesis that there is no difference between the nodes in the subgraph. Assuming the null hypothesis is true, null distributions of each test statistic were simulated by randomly sampling 183 nodes from the network 10,000 times. Using the null distributions, one-sided non-parametric $P$ values were calculated as the probability of observing a test statistic as extreme as the test statistic for the significantly altered proteins.

A subgraph of the STRING network was induced on the proteins significantly altered in AD and their neighbours and the largest connected component was selected (4842 nodes and 182,474 edges). The subgraph contained 198 of the 228 significantly altered proteins and was assessed for enrichment of Gene Ontology terms. Densely connected subgraphs were identified using $\mathrm{MCODE}^{37}$. Modules were selected with an MCODE score $>10$. As STRING is a functional interaction network, clusters of nodes may correspond to proteins from the same complex, pathway or functional family. Clusters were assessed for overrepresentation of GO-Slim terms in the Biological Process ontology using Panther ${ }^{76}$ (version 13.1) with a custom background of the 3093 proteins identified by IM-DIA-MS in healthy or A $\beta 42$ flies. Fisher's exact tests were performed and the type I error rate was controlled by correcting for multiple testing using the Benjamini-Hochberg method at a FDR of $5 \%$.

Open source software. Data analysis was performed in Python 3.6 (Python Software Foundation, https:// www.python.org) using SciPy ${ }^{77}, \mathrm{NumPy}^{78}$, Pandas ${ }^{79}$, scikit-learn ${ }^{80}$, NetworkX ${ }^{81}$, IPython $^{82}$ and Jupyter ${ }^{83}$. Figures were plotted using Matplotlib ${ }^{84}$ and seaborn.

\section{Data availability}

Raw MS data have been deposited to PRIDE.

Project accession: PXD015443.

Username: reviewer79916@ebi.ac.uk.

Password: hsOYJ8vx.

Received: 18 September 2019; Accepted: 18 August 2020

Published online: 28 October 2020

\section{References}

1. Lane, C. A., Hardy, J. \& Schott, J. M. Alzheimer's disease. Eur. J. Neurol. 25, 59-70 (2018).

2. Glenner, G. G. \& Wong, C. W. Alzheimer's disease: initial report of the purification and characterization of a novel cerebrovascular amyloid protein. Biochem. Biophys. Res. Commun. 425, 534-539 (2012).

3. Grundke-Iqbal, I. et al. Abnormal phosphorylation of the microtubule-associated protein tau (tau) in Alzheimer cytoskeletal pathology. Proc. Natl. Acad. Sci. U.S.A. 83, 4913-4917 (1986).

4. Goedert, M., Wischik, C. M., Crowther, R. A., Walker, J. E. \& Klug, A. Cloning and sequencing of the cDNA encoding a core protein of the paired helical filament of Alzheimer disease: identification as the microtubule-associated protein tau. Proc. Natl. Acad. Sci. U.S.A. 85, 4051-4055 (1988).

5. Cai, H. et al. Metabolic dysfunction in alzheimer's disease and related neurodegenerative disorders. Curr. Alzheimer Res. 9, 5-17 (2011).

6. Szutowicz, A., Bielarczyk, H., Jankowska-Kulawy, A., Pawełczyk, T. \& Ronowska, A. Acetyl-CoA the key factor for survival or death of cholinergic neurons in course of neurodegenerative diseases. Neurochem. Res. 38, 1523-1542 (2013).

7. Suberbielle, E. et al. Physiologic brain activity causes DNA double-strand breaks in neurons, with exacerbation by amyloid- $\beta$. Nat. Neurosci. 16, 613-621 (2013).

8. Raina, A. K., Monteiro, M. J., McShea, A. \& Smith, M. A. The role of cell cycle-mediated events in Alzheimer's disease. Int. J. Exp. Pathol. 80, 71-76 (1999).

9. Kanaan, N. M. et al. Axonal degeneration in Alzheimer's disease: when signaling abnormalities meet the axonal transport system. Exp. Neurol. 246, 44-53 (2013).

10. Donev, R., Kolev, M., Millet, B. \& Thome, J. Neuronal death in Alzheimer's disease and therapeutic opportunities. J. Cell. Mol. Med. 13, 4329-4348 (2009). 
11. Van Cauwenberghe, C., Van Broeckhoven, C. \& Sleegers, K. The genetic landscape of Alzheimer disease: clinical implications and perspectives. Genet. Med. 18, 421-430 (2016).

12. Zhang, Y., McLaughlin, R., Goodyer, C. \& LeBlanc, A. Selective cytotoxicity of intracellular amyloid beta peptide1-42 through p53 and Bax in cultured primary human neurons. J. Cell Biol. 156, 519-529 (2002).

13. McGowan, E. et al. Abeta42 is essential for parenchymal and vascular amyloid deposition in mice. Neuron 47, 191-199 (2005).

14. Götz, J., Chen, F., van Dorpe, J. \& Nitsch, R. M. Formation of neurofibrillary tangles in P301l tau transgenic mice induced by Abeta 42 fibrils. Science 293, 1491-1495 (2001).

15. Mullan, M. et al. A pathogenic mutation for probable Alzheimer's disease in the APP gene at the N-terminus of beta-amyloid. Nat. Genet. 1, 345-347 (1992).

16. Nilsberth, C. et al. The 'Arctic' APP mutation (E693G) causes Alzheimer's disease by enhanced Abeta protofibril formation. Nat. Neurosci. 4, 887-893 (2001).

17. Moya-Alvarado, G., Gershoni-Emek, N., Perlson, E., Bronfman, F. C. \& Neurodegeneration and Alzheimer's disease (AD). What can proteomics tell us about the Alzheimer's brain?. Mol. Cell. Proteom. 15, 409-425 (2016).

18. Lynn, B. C., Wang, J., Markesbery, W. R. \& Lovell, M. A. Quantitative changes in the mitochondrial proteome from subjects with mild cognitive impairment, early stage, and late stage Alzheimer's disease. J. Alzheimers Dis. 19, 325-339 (2010).

19. Butterfield, D. A., Di Domenico, F., Swomley, A. M., Head, E. \& Perluigi, M. Redox proteomics analysis to decipher the neurobiology of Alzheimer-like neurodegeneration: overlaps in Down's syndrome and Alzheimer's disease brain. Biochem. J. 463, 177-189 (2014).

20. Aluise, C. D. et al. Redox proteomics analysis of brains from subjects with amnestic mild cognitive impairment compared to brains from subjects with preclinical Alzheimer's disease: insights into memory loss in MCI. J. Alzheimers Dis. 23, 257-269 (2011).

21. Dammer, E. B. et al. Quantitative phosphoproteomics of Alzheimer's disease reveals cross-talk between kinases and small heat shock proteins. Proteomics 15, 508-519 (2015).

22. Sultana, R. et al. Proteomic identification of specifically carbonylated brain proteins in APP(NLh)/APP(NLh) $\times$ PS- $1(\mathrm{P} 264 \mathrm{~L}) /$ PS-1(P264L) human double mutant knock-in mice model of Alzheimer disease as a function of age. J. Proteom. 74, 2430-2440 (2011).

23. Sofola, O. et al. Inhibition of GSK-3 ameliorates A $\beta$ pathology in an adult-onset drosophila model of Alzheimer's disease. PLoS Genet. 6, e1001087 (2010).

24. Rodriguez-Suarez, E. et al. An ion mobility assisted data independent LC-MS strategy for the analysis of complex biological samples. Curr. Anal. Chem. 9, 199-211 (2013).

25. Brown, C. J., Kaufman, T., Trinidad, J. C. \& Clemmer, D. E. Proteome changes in the aging Drosophila melanogaster head. Int. J. Mass Spectrom. 425, 36-46 (2018).

26. Tain, L. S. et al. A proteomic atlas of insulin signalling reveals tissue-specific mechanisms of longevity assurance. Mol. Syst. Biol. 13, 939 (2017).

27. Anders, S. et al. Count-based differential expression analysis of RNA sequencing data using R and bioconductor. Nat. Protoc. 8, 1765-1786 (2013).

28. Zhang, Z. H. et al. A comparative study of techniques for differential expression analysis on RNA-Seq data. PLoS ONE 9, e103207 (2014).

29. Seyednasrollah, F., Laiho, A. \& Elo, L. L. Comparison of software packages for detecting differential expression in RNA-seq studies. Brief. Bioinform. 16, 59-70 (2015).

30. Robinson, M. D., McCarthy, D. J. \& Smyth, G. K. edgeR: a Bioconductor package for differential expression analysis of digital gene expression data. Bioinformatics 26, 139-140 (2010).

31. Love, M. I., Huber, W. \& Anders, S. Moderated estimation of fold change and dispersion for RNA-seq data with DESeq2. Genome Biol. 15, 550 (2014).

32. Ritchie, M. E. et al. limma powers differential expression analyses for RNA-sequencing and microarray studies. Nucleic Acids Res. 43, e47 (2015).

33. Woo, S., Leek, J. T. \& Storey, J. D. A computationally efficient modular optimal discovery procedure. Bioinformatics 27, 509-515 (2011).

34. Nueda, M. J., Tarazona, S. \& Conesa, A. Next maSigPro: updating maSigPro bioconductor package for RNA-seq time series. Bioinformatics 30, 2598-2602 (2014).

35. Szklarczyk, D. et al. The STRING database in 2017: quality-controlled protein-protein association networks, made broadly accessible. Nucleic Acids Res. 45, D362-D368 (2017).

36. Yu, H., Kim, P. M., Sprecher, E., Trifonov, V. \& Gerstein, M. The importance of bottlenecks in protein networks: correlation with gene essentiality and expression dynamics. PLoS Comput. Biol. 3, e59 (2007).

37. Bader, G. D. \& Hogue, C. W. V. An automated method for finding molecular complexes in large protein interaction networks. BMC Bioinform. 4, 2 (2003).

38. Lazar, C., Gatto, L., Ferro, M., Bruley, C. \& Burger, T. Accounting for the multiple natures of missing values in label-free quantitative proteomics data sets to compare imputation strategies. J. Proteome Res. 15, 1116-1125 (2016).

39. López-Otín, C., Blasco, M. A., Partridge, L., Serrano, M. \& Kroemer, G. The hallmarks of aging. Cell 153, 1194-1217 (2013).

40. Hou, Y. et al. Ageing as a risk factor for neurodegenerative disease. Nat. Rev. Neurol. 15, 565-581 (2019).

41. Afshordel, S., Wood, W. G., Igbavboa, U., Muller, W. E. \& Eckert, G. P. Impaired geranylgeranyltransferase-I regulation reduces membrane-associated Rho protein levels in aged mouse brain. J. Neurochem. 129, 732-742 (2014).

42. Gao, S., Yu, R. \& Zhou, X. The role of geranylgeranyltransferase i-mediated protein prenylation in the brain. Mol. Neurobiol. 53, 6925-6937 (2016).

43. D'Souza, Y., Elharram, A., Soon-Shiong, R., Andrew, R. D. \& Bennett, B. M. Characterization of Aldh2 (-/-) mice as an age-related model of cognitive impairment and Alzheimer's disease. Mol. Brain 8, 27 (2015).

44. Ohsawa, I. et al. Age-dependent neurodegeneration accompanying memory loss in transgenic mice defective in mitochondrial aldehyde dehydrogenase 2 activity. J. Neurosci. 28, 6239-6249 (2008).

45. Sade, Y. et al. IP3 accumulation and/or inositol depletion: two downstream lithium's effects that may mediate its behavioral and cellular changes. Transl. Psychiatry 6, e968 (2016).

46. Dobrin, S. E. \& Fahrbach, S. E. Rho GTPase activity in the honey bee mushroom bodies is correlated with age and foraging experience. J. Insect Physiol. 58, 228-234 (2012).

47. Owen, L. \& Sunram-Lea, S. I. Metabolic agents that enhance ATP can improve cognitive functioning: a review of the evidence for glucose, oxygen, pyruvate, creatine, and L-carnitine. Nutrients 3, 735-755 (2011).

48. Maynard, S., Fang, E. F., Scheibye-Knudsen, M., Croteau, D. L. \& Bohr, V. A. DNA damage, DNA repair, aging, and neurodegeneration. Cold Spring Harb. Perspect. Med. 5, a025130 (2015).

49. Anisimova, A. S., Alexandrov, A. I., Makarova, N. E., Gladyshev, V. N. \& Dmitriev, S. E. Protein synthesis and quality control in aging. Aging 10, 4269-4288 (2018).

50. Mattson, M. P. \& Arumugam, T. V. Hallmarks of brain aging: adaptive and pathological modification by metabolic states. Cell Metab. 27, 1176-1199 (2018).

51. Välikangas, T., Suomi, T. \& Elo, L. L. A systematic evaluation of normalization methods in quantitative label-free proteomics. Brief Bioinform. 19, 1-11 (2018). 
52. Maas, A. I. Cerebrospinal fluid enzymes in acute brain injury. 2. Relation of CSF enzyme activity to extent of brain injury. J. Neurol. Neurosurg. Psychiatry 40, 666-674 (1977).

53. Casley, C. S., Canevari, L., Land, J. M., Clark, J. B. \& Sharpe, M. A. Beta-amyloid inhibits integrated mitochondrial respiration and key enzyme activities. J. Neurochem. 80, 91-100 (2002).

54. Cardoso, S. M., Proença, M. T., Santos, S., Santana, I. \& Oliveira, C. R. Cytochrome c oxidase is decreased in Alzheimer's disease platelets. Neurobiol. Aging 25, 105-110 (2004).

55. Fukui, H., Diaz, F., Garcia, S. \& Moraes, C. T. Cytochrome c oxidase deficiency in neurons decreases both oxidative stress and amyloid formation in a mouse model of Alzheimer's disease. Proc. Natl. Acad. Sci. U.S.A. 104, 14163-14168 (2007).

56. Castellani, R. J. et al. Chitin-like polysaccharides in Alzheimer's disease brains. Curr. Alzheimer Res. 2, 419-423 (2005).

57. Kommaddi, R. P. et al. A $\beta$ mediates F-actin disassembly in dendritic spines leading to cognitive deficits in Alzheimer's disease. J. Neurosci. 38, 1085-1099 (2018).

58. $\mathrm{Hu}$, Y. et al. An integrative approach to ortholog prediction for disease-focused and other functional studies. BMC Bioinform. 12, $357(2011)$

59. Meloni, I. et al. FACL4, encoding fatty acid-CoA ligase 4, is mutated in nonspecific X-linked mental retardation. Nat. Genet. 30, 436-440 (2002).

60. Peters, H. et al. ECHS1 mutations in Leigh disease: a new inborn error of metabolism affecting valine metabolism. Brain 137, 2903-2908 (2014).

61. Datta, A., Akatsu, H., Heese, K. \& Sze, S. K. Quantitative clinical proteomic study of autopsied human infarcted brain specimens to elucidate the deregulated pathways in ischemic stroke pathology. J. Proteom. 91, 556-568 (2013).

62. McKenzie, A. T. et al. Multiscale network modeling of oligodendrocytes reveals molecular components of myelin dysregulation in Alzheimer's disease. Mol. Neurodegener. 12, 82 (2017).

63. Chi, L.-M., Wang, X. \& Nan, G.-X. In silico analyses for molecular genetic mechanism and candidate genes in patients with Alzheimer's disease. Acta Neurol. Belg. 116, 543-547 (2016).

64. Gerber, H. et al. The APMAP interactome reveals new modulators of APP processing and beta-amyloid production that are altered in Alzheimer's disease. Acta Neuropathol Commun 7, 13 (2019).

65. Terzioglu-Usak, S., Negis, Y., Karabulut, D. S., Zaim, M. \& Isik, S. Cellular model of Alzheimer's disease: A $\beta 1-42$ peptide induces amyloid deposition and a decrease in topo isomerase II $\beta$ and Nurr1 expression. Curr. Alzheimer Res. 14, 636-644 (2017).

66. Tzekov, R. et al. Sub-chronic neuropathological and biochemical changes in mouse visual system after repetitive mild traumatic brain injury. PLoS ONE 11, e0153608 (2016).

67. Kalathur, R. K. R. et al. The unfolded protein response and its potential role in Huntington's disease elucidated by a systems biology approach. F1000Research 4, 103 (2015).

68. Talwar, P. et al. Genomic convergence and network analysis approach to identify candidate genes in Alzheimer's disease. $B M C$ Genomics 15, 199 (2014).

69. Crowther, D. C. et al. Intraneuronal Abeta, non-amyloid aggregates and neurodegeneration in a Drosophila model of Alzheimer's disease. Neuroscience 132, 123-135 (2005).

70. Osterwalder, T., Yoon, K. S., White, B. H. \& Keshishian, H. A conditional tissue-specific transgene expression system using inducible GAL4. Proc. Natl. Acad. Sci. U.S.A. 98, 12596-12601 (2001).

71. Li, G.-Z. et al. Database searching and accounting of multiplexed precursor and product ion spectra from the data independent analysis of simple and complex peptide mixtures. Proteomics 9, 1696-1719 (2009).

72. Distler, U. et al. Drift time-specific collision energies enable deep-coverage data-independent acquisition proteomics. Nat. Methods 11, 167-170 (2014).

73. Silva, J. C., Gorenstein, M. V., Li, G.-Z., Vissers, J. P. C. \& Geromanos, S. J. Absolute quantification of proteins by LCMSE: a virtue of parallel MS acquisition. Mol. Cell. Proteom. 5, 144-156 (2006).

74. Bolstad, B. M., Irizarry, R. A., Astrand, M. \& Speed, T. P. A comparison of normalization methods for high density oligonucleotide array data based on variance and bias. Bioinformatics 19, 185-193 (2003).

75. Eden, E., Navon, R., Steinfeld, I., Lipson, D. \& Yakhini, Z. GOrilla: a tool for discovery and visualization of enriched GO terms in ranked gene lists. BMC Bioinform. 10, 48 (2009).

76. Mi, H., Muruganujan, A., Casagrande, J. T. \& Thomas, P. D. Large-scale gene function analysis with the PANTHER classification system. Nat. Protoc. 8, 1551-1566 (2013).

77. Oliphant, T. E. SciPy: Open source scientific tools for Python. Computing in Science and Engineering 9, 10-20 (2007).

78. Oliphant, T. E. A guide to NumPy. vol. 1 (Trelgol Publishing USA, 2006).

79. McKinney, W. \& Others. Data structures for statistical computing in python. in Proceedings of the 9th Python in Science Conference vol. 445, 51-56 (Austin, TX, 2010).

80. Pedregosa, F. et al. Scikit-learn: machine learning in Python. J. Mach. Learn. Res. 12, 2825-2830 (2011).

81. Hagberg, A., Swart, P. \& S Chult, D. Exploring Network Structure, Dynamics, and Function Using NetworkX. https://www.osti.gov/ biblio/960616 (2008).

82. Perez, F. \& Granger, B. E. IPython: A System for Interactive Scientific Computing. Computing in Science Engineering 9, 21-29 (2007).

83. Kluyver, T. et al. Jupyter Notebooks-a publishing format for reproducible computational workflows. in ELPUB 87-90 (2016).

84. Hunter, J. D. Matplotlib: A 2D graphics environment. Comput. Sci. Eng. 9, 90-95 (2007).

\section{Acknowledgements}

HS is supported by an ISMB Wellcome PhD studentship [203780/Z/16/A]; AC is supported by a BBSRC CASE $\mathrm{PhD}$ studentship and Waters Corporation; JL is supported by BBSRC [BB/L002817/1]; LP and FK are supported by a Wellcome Trust Strategic Award to LP [098565/Z/12/Z] and an Alzheimer's Research UK Project Grant [ART-2009-4]. The work was supported by a Wellcome instrumentation Grant [104913/Z/14/Z]. We thank Dr Damian Crowther (University of Cambridge) for donation of UAS-A $\beta 42$ fly stocks and Dr Hervé Tricoire (CNRS, France) for donation of elavGS fly stocks. Fig. 1c: fly graphic by Daan Kauwenberg, from the Noun Project. We also thank Thomas Menneteau for assisting with raw data upload to PRIDE.

\section{Author contributions}

A.C., F.K., L.A.G. performed experiments. H.M.S., A.C., D.S., J.G.L., J.P.C.V. analysed data under the supervision of C.A.O., L.P. and K.T. The manuscript was written by H.M.S., A.C., F.K. with input from C.A.O., L.P., K.T. C.A.O., L.P. and K.T. acquired funds to carry out the research. The study was designed by A.C., H.M.S., F.K., C.A.O., L.P. and K.T. The project was managed by K.T. All authors edited, reviewed and approved the final manuscript. 


\section{Competing interests}

The authors declare no competing interests.

\section{Additional information}

Supplementary information is available for this paper at https://doi.org/10.1038/s41598-020-74748-9.

Correspondence and requests for materials should be addressed to C.A.O., L.P. or K.T.

Reprints and permissions information is available at www.nature.com/reprints.

Publisher's note Springer Nature remains neutral with regard to jurisdictional claims in published maps and institutional affiliations.

Open Access This article is licensed under a Creative Commons Attribution 4.0 International License, which permits use, sharing, adaptation, distribution and reproduction in any medium or format, as long as you give appropriate credit to the original author(s) and the source, provide a link to the Creative Commons licence, and indicate if changes were made. The images or other third party material in this article are included in the article's Creative Commons licence, unless indicated otherwise in a credit line to the material. If material is not included in the article's Creative Commons licence and your intended use is not permitted by statutory regulation or exceeds the permitted use, you will need to obtain permission directly from the copyright holder. To view a copy of this licence, visit http://creativecommons.org/licenses/by/4.0/.

(C) The Author(s) 2020 